\title{
Physics opportunities with a fixed target experiment at the LHC (AFTER@LHC)
}

\author{
C. Hadjidakis ${ }^{* 1}$, M. Anselmino ${ }^{2}$, R. Arnaldi ${ }^{2}$, S.J. Brodsky ${ }^{3}$, V. Chambert ${ }^{2}$, C. Da \\ Silva $^{4}$, J.P. Didelez ${ }^{1}$, M.G. Echevarria年, E.G. Ferreiro' ${ }^{6}$, F. Fleuret ${ }^{7}$, Y. Gao ${ }^{8}$, \\ B. Genolini ${ }^{1}$, I. Hřivnáčová ${ }^{1}$, D. Kikola ${ }^{9}$, A. Klein ${ }^{4}$, A. Kurepin ${ }^{10}$, A. Kusina ${ }^{11}$, \\ J.P. Lansberg ${ }^{1}$, C. Lorcé ${ }^{12}$, F. Lyonnet ${ }^{13}$, G. Martinez ${ }^{14}$, L. Massacrier ${ }^{1}$, A. Nass ${ }^{15}$, \\ C. Pisano ${ }^{16}$, P. Robbe ${ }^{17}$, I. Schienbein ${ }^{18}$, M. Schlegel ${ }^{19}$, E. Scomparin ${ }^{2}$, J. Seixas ${ }^{20}$, \\ H.S. Shao ${ }^{21}$, A. Signori' ${ }^{22}$, E. Steffens ${ }^{23}$, L. Szymanowski ${ }^{24}$, N. Topilskaya ${ }^{10}$, \\ B. Trzeciak $^{25}$, U.I. Uggerhøj ${ }^{26}$, A. Uras ${ }^{27}$, R. Ulrich ${ }^{28}$, J. Wagner ${ }^{24}$, N. Yamanaka ${ }^{1}$, \\ Z. Yang ${ }^{8}$
}

${ }^{1}$ IPNO, CNRS/IN2P3, Univ. Paris-Sud, Université Paris-Saclay, Orsay, France

${ }^{2}$ Dip. di Fisica and INFN Sez. Torino, Via P. Giuria 1, Torino, Italy

${ }^{3}$ SLAC National Accelerator Laboratory, Stanford University, Menlo Park, USA

${ }^{4}$ LANL, P-25, Los Alamos National Laboratory, Los Alamos, NM 87545, USA

${ }^{5}$ INFN Sez. Pavia, Via Bassi 6, 27100 Pavia, Italy

${ }^{6}$ Dept. de Física de Partículas, USC, Santiago de Compostella, Spain

${ }^{7}$ LLR, École Polytechnique, CNRS/IN2P3, Palaiseau, France

${ }^{8}$ CHEP, Department of Engineering Physics, Tsinghua University, Beijing, China

${ }^{9}$ Faculty of Physics, Warsaw University of Technology, Warsaw, Poland

${ }^{10}$ Institute for Nuclear Research, Russian Academy of Sciences, Moscow, Russia

${ }^{11}$ Institute of Nuclear Physics Polish Academy of Sciences, PL-31342 Krakow, Poland

${ }^{12}$ CPhT, Ecole Polytechnique, CNRS, Université Paris-Saclay, Palaiseau, France

${ }^{13}$ Southern Methodist University, Dallas, TX 75275, USA

${ }^{14}$ SUBATECH, IMT Atlantique, Université de Nantes, CNRS-IN2P3, Nantes, France

15 Institut für Kernphysik, Forschungszentrum Jülich, Jülich, Germany

${ }^{16}$ Dipartimento di Fisica, Universita degli Studi di Pavia, Pavia, Italy

${ }_{17}$ LAL, Univ. Paris-Sud, CNRS/IN2P3, Université Paris-Saclay, Orsay, France

${ }_{18}$ LPSC, Université Grenoble-Alpes, CNRS/IN2P3, 38026 Grenoble, France

${ }^{19}$ Institute for Theoretical Physics, Tübingen U., Tübingen, Germany

${ }^{20}$ LIP and IST, Lisbon, Portugal

${ }^{21}$ Laboratoire de Physique Théorique et Hautes Énergies (LPTHE), UMR 7589, Sorbonne Université et CNRS, 4 place Jussieu, 75252 Paris Cedex 05, France

22 Theory Center, Thomas Jefferson National Accelerator Facility, 12000 Jefferson Avenue, Newport News, VA 23606, USA

${ }^{23}$ Physics Institute, Friedrich-Alexander University Erlangen-Nürnberg, Erlangen, Germany

${ }^{24}$ National Centre for Nuclear Research (NCBJ), Hoza 69, 00-681, Warsaw, Poland

25 Institute for Subatomic Physics, Utrecht University, Utrecht, The Netherlands

${ }^{26}$ Department of Physics and Astronomy, University of Aarhus, Denmark

${ }^{27}$ IPNL, Université Claude Bernard Lyon-I, CNRS/IN2P3, Villeurbanne, France

28 Institut für Kernphysik, Karlsruhe Institute of Technology (KIT), Karlsruhe, Germany

By extracting the beam with a bent crystal or by using an internal gas target, the multi-TeV proton and lead LHC beams allow one to perform the most energetic fixed-target experiments (AFTER@LHC) and to study $p+p$ and $p+\mathrm{A}$ collisions at $\sqrt{s_{N N}}=115 \mathrm{GeV}$ and $\mathrm{Pb}+p$ and $\mathrm{Pb}+\mathrm{A}$ collisions at $\sqrt{s_{N N}}=72 \mathrm{GeV}$. Such studies would address open questions in the domain of the nucleon and nucleus partonic structure at high- $x$, quark-gluon plasma and, by using longitudinally or transversally polarised targets, spin physics. In this paper, we discuss the physics opportunities of a fixed-target experiment at the LHC and we report on the possible technical implementations of a high-luminosity experiment. We finally present feasibility studies for Drell-Yan, open heavyflavour and quarkonium production, with an emphasis on high- $x$ and spin physics.

XVII International Workshop on Polarized Sources, Targets \& Polarimetry

16-20 October 2017

Kaist, South Korea

* Speaker. 


\section{Introduction}

Fixed-target experiments present many advantages having the versatility of polarised and nuclear targets and allowing to reach high-luminosity with dense and long target. The AFTER@LHC project aims at demonstrating the physics opportunities and the technical implementations of a high-luminosity fixed-target experiment using the LHC beams. The $7 \mathrm{TeV}$ proton and $2.76 \mathrm{~A}$ $\mathrm{TeV}$ lead beams allow to reach a center-of-mass energy per nucleon pair of $\sqrt{s_{N N}}=115 \mathrm{GeV}$ and $\sqrt{s_{N N}}=72 \mathrm{GeV}$ with a center-of-mass rapidity boost of 4.8 and 4.2 units, respectively. These energies correspond to an energy domain between SPS and nominal RHIC energies. The large rapidity boost implies that the mid- to forward rapidity region in the center of mass frame $\left(y_{c m s} \geq 0\right)$ lies within 1 degree in the laboratory frame and that the backward rapidity region $\left(y_{c m s} \leq 0\right)$ is easily accessible by using standard experimental techniques or existing LHC experiments such as ALICE or LHCb. Thus the fixed-target mode at high-luminosity offers unique opportunities to access the very backward rapidity domain and therefore the high- $x$ frontier, where $x$ is the momentum fraction of the parton struck in the target nucleon or nucleus.

The physics motivations of a fixed-target experiment at the LHC are three-fold: advance our understanding of the high- $x$ gluon, antiquark and heavy-quark content in the nucleon and nucleus, unravel the spin structure of the nucleon and study the quark-gluon plasma (QGP) created in heavyion collisions [1, 2, 3, 4] (see also [5] for more details). In the high- $x$ programme, one can extract parton distribution functions (PDFs), poorly known in this kinematic regime, by using for instance Drell-Yan measurements to probe the light sea quark and open heavy-flavour measurements to probe the gluon content of the nucleon. One should emphasize that at large- $x$, intrinsic charm could also enhance the open heavy-flavour and charmonium production [6]. This is particularly relevant for high-energy neutrino and cosmic ray physics. In a nuclear target, one could reveal the EMC effect which is far to be understood and determine whether such an effect exists also for the gluon case. With a transversally polarised target, one could access information on orbital motion of partons bound into hadrons by measuring the Single Transverse Spin Asymmetries (STSA): a Sivers effect is expected to be important in particular at large $x$ as it will be discussed in the following. This effect can be probed for light sea quark with Drell-Yan or gluon with open heavyflavour production for instance. In particular, a sign change of the asymmetry is predicted for Drell-Yan production with respect to semi-inclusive production in deep inelastic scattering (DIS). Also spin physics can be carried out with an unpolarised target by studying the Boer-Mulder effect and determining linearly polarised gluons in an unpolarised proton. Finally in $\mathrm{Pb}-\mathrm{A}$ collisions at $\sqrt{s_{N N}}=72 \mathrm{GeV}$, the medium created is expected to have a low baryon chemical potential and a temperature approximately 1.5 times higher than the critical temperature of the phase transition between a hadron gas and a QGP [7]. At such temperature, the excited states of $\Upsilon$ are expected to melt into the QGP [8]. In addition, measurements of particle yields and their anisotropies as a function of rapidity and system size would allow one to scan the phase-transition region. In the following, we will concentrate on the high- $x$ and spin physics topics.

\section{Technical implementations}

There are various techniques to obtain a fixed-target experiment in a collider that are or were 
already used in current and past experiments. An internal gaseous target is currently used at RHIC (gas-jet from atomic beam source [9]) for measuring the proton beam polarisation and was also used by HERMES at HERA (atomic beam source associated with a storage cell [10]). At the LHC, LHCb has pioneered the use of gaseous fixed-target with the SMOG system [11, 12], originally designed for luminosity calibration. In that case, the gas density is low since the gas is not confined to a specific region and there is no dedicated pumping system. Also only noble gases have been used so far and for limited running time periods. In the gas target case, the full LHC proton or lead flux is used. The gas-jet [13] and storage cell [14] setups can provide a target areal density as high as $10^{15}-10^{16} \mathrm{~cm}^{-2}$ with unpolarised hydrogen, i.e. an increase by three to four order of magnitude with respect to SMOG. The atomic beam source can inject polarised gas such as $H^{\uparrow}$, $D^{\uparrow}$ and ${ }^{3} \mathrm{He}^{\uparrow}$. For polarised hydrogen, the target areal density is of the order of $10^{14} \mathrm{~cm}^{-2}$ for the storage cell solution, higher by two order of magnitude than for the gas-jet.

\begin{tabular}{c|c|c|c} 
Technique & System & $\mathscr{L}\left[\mathrm{cm}^{-2} \mathrm{~s}^{-1}\right]$ & $\mathscr{L}_{\text {int }}$ \\
\hline Gas jet & $p+\mathrm{H}^{\uparrow}$ & $4.5 \cdot 10^{30}$ & $45 \mathrm{pb}^{-1}$ \\
& $p+\mathrm{H}_{2}$ & $\sim 10^{33}-10^{34}$ & $\sim 10-100 \mathrm{fb}^{-1}$ \\
\hline Storage cell & $p+\mathrm{H}^{\uparrow}$ & $0.9 \cdot 10^{33}$ & $9 \mathrm{fb}^{-1}$ \\
& $p+\mathrm{H}_{2}$ & $5.8 \cdot 10^{33}$ & $58 \mathrm{fb}^{-1}$ \\
& $\mathrm{~Pb}+\mathrm{Xe}$ & $3 \cdot 10^{28}$ & $30 \mathrm{nb}^{-1}$ \\
\hline Bent crystal & $p+\mathrm{H}$ & $1.3 \cdot 10^{31}$ & $130 \mathrm{pb}^{-1}$ \\
and 5 mm thick target & $p+\mathrm{W}$ & $1.6 \cdot 10^{31}$ & $160 \mathrm{pb}^{-1}$ \\
& $\mathrm{~Pb}+\mathrm{W}$ & $3 \cdot 10^{27}$ & $3 \mathrm{nb}^{-1}$ \\
\hline
\end{tabular}

Table 1: Instantaneous and yearly integrated luminosity for few systems for the gas jet, storage cell and bent crystal techniques (see text). The integrated luminosity corresponds to a LHC year with time duration of $t_{p}=10^{6} \mathrm{~s}$ and $t_{\mathrm{Pb}}=10^{7} \mathrm{~s}$ for the proton and lead beams, respectively.

Another option to obtain a fixed-target is the use of a solid target located inside the beam pipe as currently used by STAR at RHIC for the beam energy scan programme [15] or used in the past by HERA-B [16]. In that case, the beam halo interacts directly with the target. Another more promising solution, that has the advantage to be more parasitic to the main beams, is to deviate the beam halo on an internal solid target by using a bent crystal [17]. In that case, the proton and lead fluxes are expected to be approximately $5.10^{8} \mathrm{p} / \mathrm{s}$ and $2.10^{5} \mathrm{~Pb} / \mathrm{s}$ and would allow to obtain large luminosities with a target of few millimeter thickness. With this technique, two solutions can be envisioned: either the beam is extracted and a new beam line is created, or the beam is used on a target located in an existing cavern (beam splitting). The first solution requires however civil engineering and could be achieved only over a long timescale. The second solution is possible providing that the deflected beam halo is then absorbed upstream of the detector.

Table 1 presents the instantaneous and integrated luminosity per LHC year for the gaseous target solutions in the case of the gas-jet and storage cell as well as for the bent crystal associated with a solid target. Few possible target types are shown. Instantaneous luminosities up to $0.9 \cdot 10^{33}$ $\mathrm{cm}^{-2} \mathrm{~s}^{-1}$ can be obtained with the storage cell technique and a polarised hydrogen gas. If running the full LHC year, this translates to an integrated luminosity of $9 \mathrm{fb}^{-1}$. In the case of polarised targets, the beam fraction used over a fill is always lower than a percent. It can reach few percents with proton beam on $\mathrm{H}_{2}$ target and up to $15 \%$ for $\mathrm{Pb}+\mathrm{Xe}$. For the physics with polarised target, 
the spin figure of merit can be derived according to $\mathscr{F}=\mathscr{P}_{\text {eff }} \times \mathscr{L}$ with $\mathscr{L}$ the instantaneous luminosity. The effective polarisation is defined as $\mathscr{P}_{\text {eff }}=p_{T}^{2} \times f^{2}$ with $p_{T}$ and $f$, the polarisation and dilution factor of the target, respectively. While the spin figure of merit is $\mathscr{F}=3.4 \cdot 10^{30}$ $\mathrm{cm}^{-2} \mathrm{~s}^{-1}$ for the gas-jet, it reaches $\mathscr{F}=6.4 \cdot 10^{32} \mathrm{~cm}^{-2} \mathrm{~s}^{-1}$ for the storage cell.

The ALICE and LHCb detectors are well suited to set up a fixed-target programme at the LHC. The ALICE detector with its forward muon arm and future Muon Forward Tracker can measure muons with a center-of-mass rapidity coverage ranging from mid- to backward rapidity. The central barrel of ALICE can provide measurements at very backward rapidity in the center of mass with excellent particle identification (PID) capabilities. The LHCb detector is fully instrumented in the forward region in the laboratory frame, with vertexing, tracking and triggering detector systems, excellent PID and high data-acquisition rate capabilities. In the projected performances discussed in the next section, we will assume a LHCb-like detector setup and an integrated luminosities of $\mathscr{L}_{\text {int }}=10 \mathrm{fb}^{-1}$ in $p+p$ collisions at $\sqrt{s}=115 \mathrm{GeV}$. This luminosity can be collected in a year and corresponds to an instantaneous luminosity of $\mathscr{L}=10^{33} \mathrm{~cm}^{-2} \mathrm{~s}^{-1}$ and an inelastic rate of $39 \mathrm{MHz}$ by considering a cross-section of $\sigma_{\text {inel }}=39 \mathrm{mb}$.

\section{Projected performances}

The partonic structure of the nucleon can be studied with Drell-Yan in $p+p$ collisions. In particular AFTER@LHC will allow to complement the existing Drell-Yan measurements by covering the large $x$ domain up to values close to 1 . The left panel of Fig. 1 shows the Drell-Yan lepton pair production cross-section as a function of the muon pair center-of-mass rapidity from pseudo-data in $p+p$ collisions at $\sqrt{s}=115 \mathrm{GeV}$ for $\mathscr{L}_{\text {int }}=10 \mathrm{fb}^{-1}$. The pseudo-data follow the NLO theory predictions obtained using CT14 PDFs. The projected experimental uncertainties are estimated from a fast simulation setup of a LHCb-like detector as described in [2, 4]. The single muon tracking and the identification efficiency is $\sim 98 \%$, the kinematic acceptance is $2<\eta<5$ and a $p_{T}$ cut is set at $p_{T}>1.2 \mathrm{GeV} / c$. In addition, the dimuon kinematic fulfills the following requirements: $2<y_{\mu \mu}<5$ and $4 \leq M_{\mu \mu} \leq 5 \mathrm{GeV} / c^{2}$. The band, that represents the theoretical uncertainties, is much larger than the projected statistical precision. A profiling analysis has been performed to evaluate the impact of the Drell-Yan measurements on the PDFs and the right panel of Fig. 1 illustrates the result for the $u$ quark PDF uncertainties shown as a function of $x$ at a scale of $Q=1.3 \mathrm{GeV}$. The valence as well as the light sea quark PDFs can be largely constrained with these measurements over a large $x$ range. In particular, the valence quark uncertainty can be considerably reduced for $x>0.4$ as illustrated for the $u$ quark. In $p+A$ collisions, the existing Drell-Yan measurements are scarce and AFTER @LHC will contribute to reduce the uncertainties on the nuclear PDF (nPDF), in particular at large $x$.

By using open heavy-flavour production such as $\mathrm{D}$ mesons, one can probe the gluon and charm content of the nucleon. In particular the charm PDF uncertainties are quite large for $x>0.1$ and it is a longstanding debate whether the charm contribution of the nucleon arises from a perturbative component via gluon splitting or a non-perturbative one from intrinsic charm. Such studies are also relevant for cosmic ray physics since an intrinsic charm component would result into a modification of the neutrino production cross-section. The left panel of Fig. 2 shows the projected uncertainties for the $D^{0}$ meson yield in $p+p$ collisions at $\sqrt{s}=115 \mathrm{GeV}$ as a function of $D^{0}$ meson $p_{T}$ in three 

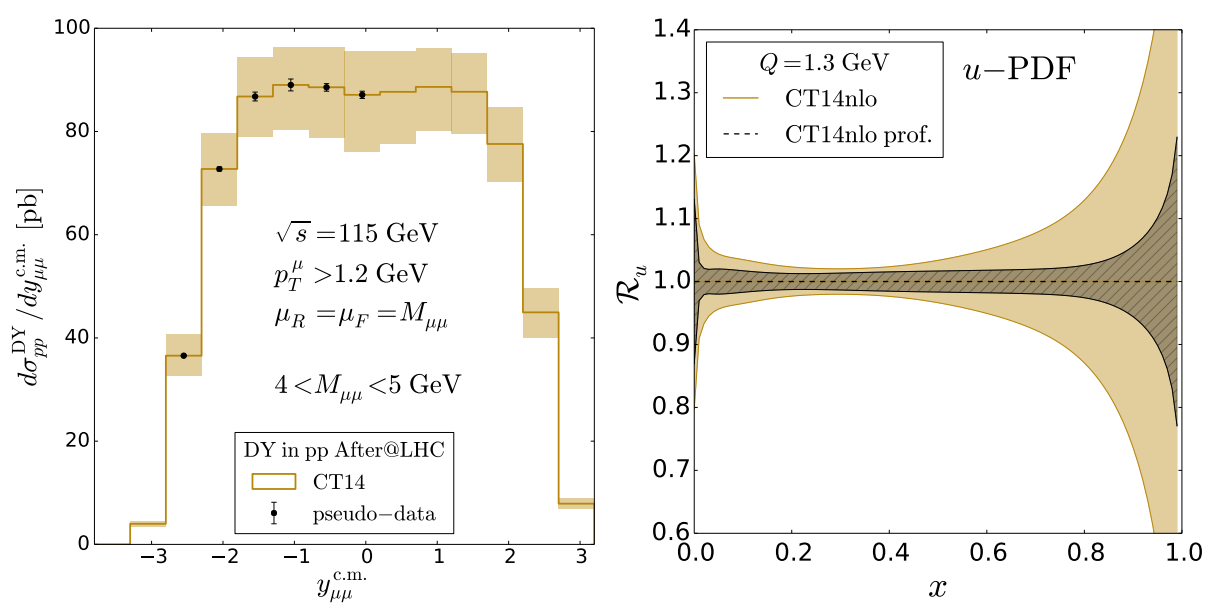

Figure 1: Left panel: Drell-Yan lepton pair production cross-section in $p+p$ collisions at $\sqrt{s}=115 \mathrm{GeV}$ as a function of the muon pair rapidity for $4 \leq M_{\mu \mu} \leq 5 \mathrm{GeV} / c^{2}$ from pseudo-data with a LHCb-like detector. The NLO theory predictions are obtained using CT14 PDFs. Right panel: Impact of the Drell-Yan lepton pair pseudo-data on the $u$ quark PDF uncertainties as a function of $x$ at a scale $Q=1.3 \mathrm{GeV}$.
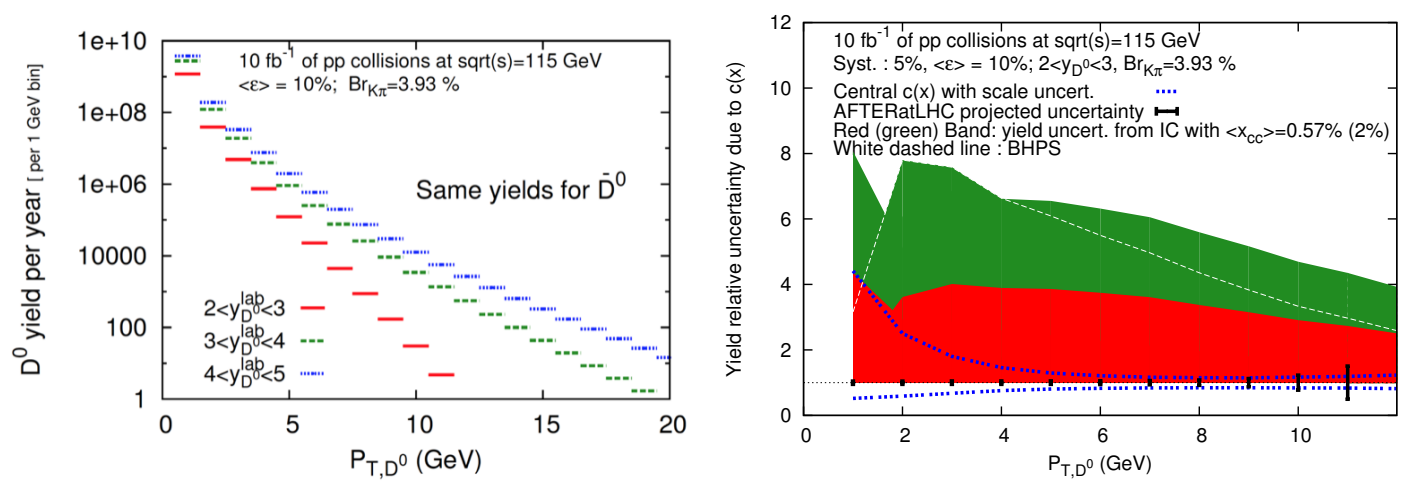

Figure 2: Left panel: Projected $D^{0}$ meson yield in $p+p$ collisions at $\sqrt{s}=115 \mathrm{GeV}$ as a function of $p_{T}$ with a LHCb-like detector. Right panel: Relative uncertainties on the $D^{0}$ meson yield arising from the charm content of the proton compared to projected total uncertainties from pseudo-data.

rapidity bins. On the right panel, the relative uncertainties from the simulations are compared to the theoretical uncertainties obtained using the setup described in [6]. The expected precision on the D meson yield down to low $p_{T}$ will allow to set strong constraints on theoretical models.

With transversally polarised target, the Drell-Yan process can probe the quark Sivers Transverse Momentum Dependent (TMD) function, related to its orbital angular momentum. By using a storage cell, a high spin figure of merit and precise measurements are expected as previously discussed. The STSA for pseudo-data in $p+p^{\uparrow}$ collisions at $\sqrt{s}=115 \mathrm{GeV}$ with $\mathscr{L}_{\text {int }}=10 \mathrm{fb}^{-1}$ are shown on the left panel of Fig. 3. An effective polarisation of $80 \%$ is assumed for the transversally polarised hydrogen target. Theoretical calculations from [18] are also displayed. The asymmetry is predicted to be larger at large $x^{\uparrow}$. The large statistical precision and the wide rapidity coverage of the Drell-Yan production in this experimental setup [4] will allow to probe the $x$ dependence of the STSA and to constrain model calculations. In addition, the Drell-Yan process can be measured 
with a polarised ${ }^{3} \mathrm{He}$ target. The right panel of Fig. 3 shows the projected statistical precision for the Drell-Yan STSA in $p+{ }^{3} \mathrm{He}^{\uparrow}$ collisions at $\sqrt{s}=115 \mathrm{GeV}$ for $\mathscr{L}_{\text {int }}=2.5 \mathrm{fb}^{-1}$. In that case the effective polarisation is diluted by a factor of three since only the neutron is polarised. This measurement will allow to access the quark Sivers function in a polarised neutron.
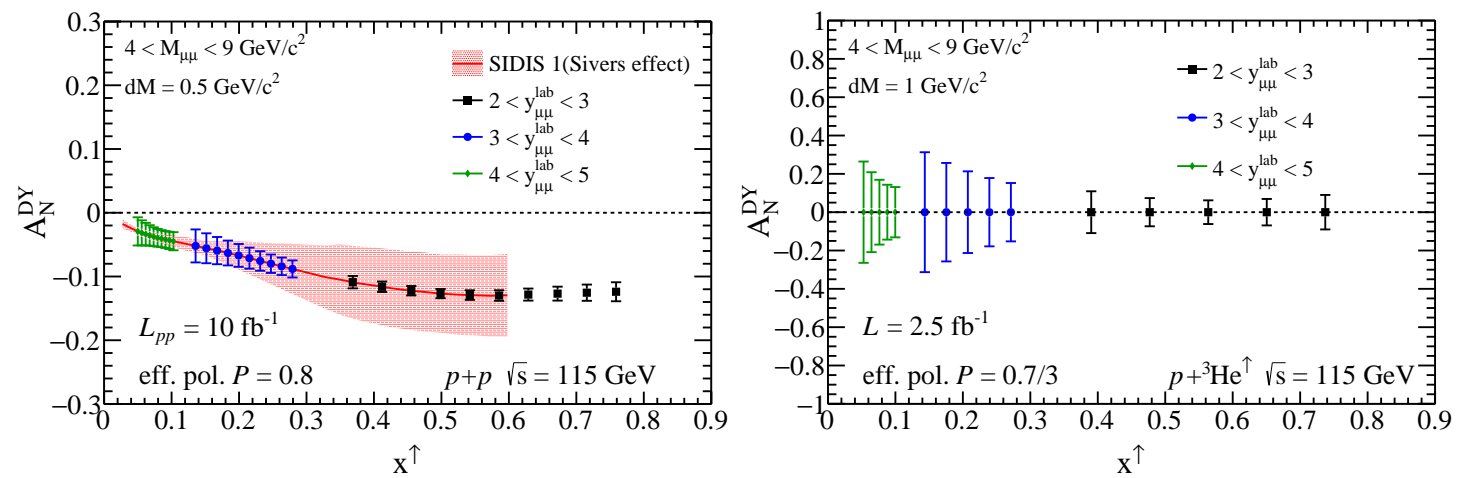

Figure 3: Left panel: Drell-Yan $A_{N}$ in $p+p^{\uparrow}$ collisions at $\sqrt{s}=115 \mathrm{GeV}$ as a function of the parton momentum fraction $x^{\uparrow}$ [18] compared to the estimated statistical precision of the measurement with a LHCb-like detector. Right panel: Statistical projections for Drell-Yan $A_{N}$ as a function of $x^{\uparrow}$ in $p+{ }^{3} \mathrm{He}^{\uparrow}$ collisions.

The open charm and quarkonium measurements will complement the Drell-Yan measurements with a transversally polarised target. Since open heavy-flavour and quarkonium production are sensitive to the gluon content of the nucleon, the STSA measurements will be useful to probe the gluon Sivers effect, which is essentially unknown. Such a measurement does not exist so far for the $\mathrm{D}$ meson and only data from RHIC exist for the $\mathrm{J} / \psi$ with however large uncertainties. The projected uncertainties for the STSA asymmetries are shown on the left and right panel of Fig. 4 for the $D^{0}$ meson and charmonium production, respectively. More details on simulation inputs are given in $[4,19]$. The Feynman $x$ variable is defined as $x_{F}=x_{1}-x_{2}$ with $x_{1}\left(x_{2}\right)$ the parton momentum fraction probed in the projectile (target). With the expected yields, these measurements will be essentially limited by the systematic uncertainties and will give a precise access to the gluon content of the proton.
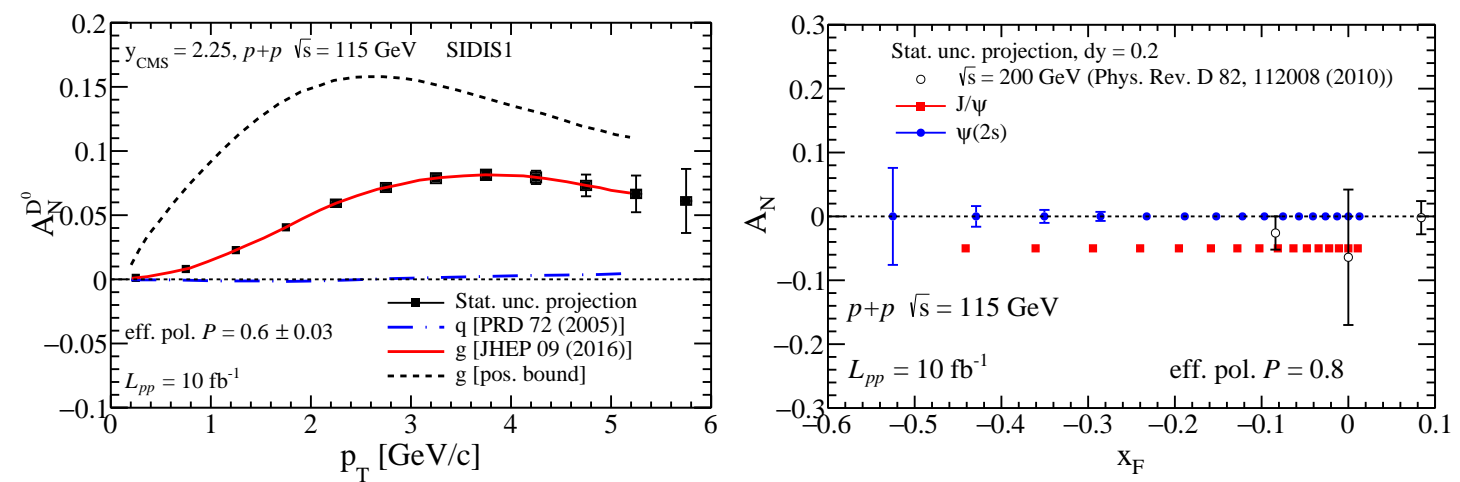

Figure 4: Left panel: Projections for $D^{0}$ meson $A_{N}$ in $p+p^{\uparrow}$ collisions at $\sqrt{s}=115 \mathrm{GeV}$ as a function of $p_{T}$ with a LHCb-like detector. Right panel: Projections for charmonium $A_{N}$ as a function of $x_{F}$ and compared to RHIC measurement. 


\section{Conclusion}

Many physics opportunities are offered by a high-luminosity fixed-target experiment at the LHC with a detector covering a wide rapidity range. Various implementations are possible and are currently under investigation at the LHC. The achievable luminosities are promising for decisive measurements both on quark and gluon sensitive probes such as Drell-Yan, open heavy-flavour and quarkonium production. With unpolarised targets, the Drell-Yan measurements will contribute to reduce the uncertainties on the Parton Distribution Function in the nucleon and nucleus, in particular at large $x$. In addition, the expected precision on the D meson yields will set strong constraints on the proton intrinsic charm content. With transversally polarised targets, it will be possible to study the quark and gluon Sivers functions by measuring the single transverse spin asymmetries for Drell-Yan, open and hidden heavy-flavour production.

Acknowledgement This research was supported by the French P2IO Excellence Laboratory, the French CNRS via the grants FCPP-Quarkonium4AFTER \& Défi Inphyniti-Théorie LHC France, the P2I department of the Paris-Saclay University and by the COPIN-IN2P3 Agreement. AS acknowledges support from U.S. Department of Energy contract DE-AC05-06OR23177, under which Jefferson Science Associates, LLC, manages and operates Jefferson Lab.

\section{References}

[1] S. J. Brodsky, F. Fleuret, C. Hadjidakis and J. P. Lansberg, Phys. Rept. 522 (2013) 239

[2] L. Massacrier et al.,Adv. High Energy Phys. 2015 (2015) 986348

[3] B. Trzeciak et al., Few Body Syst. 58 (2017) no.5, 148

[4] D. Kikola et al., Few Body Syst. 58 (2017) no.4, 139

[5] http://after.in2p3.fr

[6] S. J. Brodsky et al.,Adv. High Energy Phys. 2015 (2015) 231547

[7] H. Satz, J. Phys. G 32 (2006) R25

[8] A. Mocsy, P. Petreczky and M. Strickland, Int. J. Mod. Phys. A 28 (2013) 1340012

[9] A. Zelenski et al., Nucl. Instrum. Meth. A 536 (2005) 248.

[10] A. Airapetian et al. [HERMES Collaboration], Nucl. Instrum. Meth. A 540 (2005) 68

[11] R. Aaij et al. [LHCb Collaboration], JINST 9 (2014) no.12, P12005

[12] The LHCb Collaboration,LHCb-CONF-2017-001, CERN-LHCb-CONF-2017-001.

[13] A. Zelenski, private communication

[14] C. Barschel, P. Lenisa, A. Nass and E. Steffens, Adv. High Energy Phys. 2015 (2015) 463141.

[15] K. C. Meehan [STAR Collaboration], J. Phys. Conf. Ser. 742 (2016) no.1, 012022.

[16] C. Hast et al., Nucl. Instrum. Meth. A 354 (1995) 224.

[17] W. Scandale et al., Phys. Lett. B 758 (2016) 129.

[18] M. Anselmino, U. D’ Alesio and S. Melis, Adv. High Energy Phys. 2015 (2015) 475040

[19] D. Kikola, Adv. High Energy Phys. 2015 (2015) 783134. 